\title{
Comparison of Endosperm Amyloplast Development and Degradation in Waxy and Non-waxy Wheat
}

\author{
H. Yu, Y. YAnG, X.Y. Chen, G.X. Lin, J.Y. Sheng, J.Y. Nie, Q.J. WANG, E.J. Zhang, X.R. Yu, \\ Z. WANG and F. XIONG*
}

Jiangsu Key Laboratory of Crop Genetics and Physiology/Co-Innovation Center for Modern Production Technology of Grain Crops/Joint International Research Laboratory of Agriculture \& Agri-Product Safety, Yangzhou University, Yangzhou, China

(Received 29 January 2017; Accepted 24 April 2017;

Communicated by F. Békés)

\begin{abstract}
The waxy wheat shows special starch quality due to high amylopectin content. However, little information is available concerning the development and degradation of amyloplast from waxy wheat endosperm. To address this problem, waxy wheat variety, Yangnuo 1, and a non-waxy wheat variety, Yangmai 13, were chosen to investigate the development and degradation of endosperm amyloplast during wheat caryopsis development and germination stage respectively using histochemical staining and light microscopy. Changes of morphology, the soluble sugar and total starch content were indistinguishable in the process of caryopsis development of two wheat varieties. The developing endosperm of non-waxy was stained blue-black by $\mathrm{I}_{2}-\mathrm{KI}$ while the endosperm of waxy wheat was stained reddish-brown, but the pericarp of waxy and non-waxy wheat was stained blue-black. In contrast to nonwaxy wheat, endosperm amyloplast of waxy wheat had better development status and higher proportion of small amyloplast. During seed germination many small dissolution pores appeared on the surface of endosperm amyloplast and the pores became bigger and deeper until amyloplast disintegrated. The rate of degradation of waxy wheat endosperm amyloplast was faster than non-waxy wheat. Our results may also be helpful to the use of waxy starch in food and nonfood industry.
\end{abstract}

Keywords: waxy wheat, amyloplast, development, degradation

\section{Introduction}

Wheat (Triticum aestivum L.), one of the most important grain crops, is widely cultivated around the world. As one of the three main food crops, wheat played a vital role in the development of human civilization (Dodson et al. 2013). As the most important reserve substances in wheat, starch accounts for about 50-60\% of the grain dry weight (Wang et al. 2015) and exists in wheat endosperm in the form of amyloplast. Starch was constituted by amylose and amylopectin, and the content of amylose from $22-25 \%$ in normal wheat (Morrison et al. 1984). The synthesis of amylose was controlled by granule-bound starch synthase (GBSS, EC24.1.21), which was composed of three subunits. In normal wheat, 
the three subunits were controlled by three homoeologous waxy genes, $W x-A 1, W x-B 1$ and $W x-D 1$, respectively (Chao et al. 1989). The deficient of one or two waxy genes created partially waxy plant and the waxy plant with three non-functional waxy genes (Ma et al. 2013). Therefore, the pattern changes of starch synthase in waxy plant had significant effect on amyloplast and the structure of starch (Macdonald and Preiss 1985). In 1995, waxy wheat was first cultivated by artificial hybridization (Nakamura et al. 1995). In recent years, scientists in Australia, Canada, the United States, China and others had researched on waxy wheat (Zhang et al. 2013; Ma et al. 2013).

Since the extremely high content of amylopectin, waxy wheat starch exhibits some special physicochemical properties. Waxy wheat starch has lower pasting temperature, lower setback viscosity, higher peak viscosity values, rapid swelling and higher thermal stability (Sasaki et al. 2000). A lot of researches on the application of waxy wheat starch also have been done. Noodles made by waxy wheat starch had higher taste quality (Baik and Lee 2003). The application of waxy wheat starch was good for slowing down bread staling for the reason of the lower pasting temperature and lower setback viscosity (Sasaki et al. 2000). Overall, waxy wheat starch has important application value and high economic value in starch industry, food processing industry and the development of special food (Morell et al. 1995).

The development of waxy wheat plays an important role in the structure and properties of starch, thus affects the quality and application of starch (Mira et al. 2007). According to the shape and size, endosperm amyloplast could be classified as A- $(\geq 10 \mu \mathrm{m}$ in diameter) and B-type ( $<10 \mu \mathrm{m}$ in diameter) (Parker 1985). The A- and B-type amyloplast have different amylose, amylopectin, lipid, and protein content. The differences in chemical composition will influence on crystallinity, swelling properties, pasting properties and other physicochemical properties of starch (Hung et al. 2006; Li et al. 2013). However, there is a small amount of information about the proportion of A- and B-type amyloplast in waxy and non-waxy wheat.

Seed germination is a complex and order process, which involves different tissues and many substances. As the main storage place of nutrients, endosperm amyloplast provided important energy and substances for germinating seed in the early growth stage (Van Dongen et al. 2004). The degradation of endosperm amyloplast associated with the synthesis and secretion of relevant hydrolytic enzymes controlled by the embryo, scutellum, and aleurone layer cells (Domínguez and Cejudo 2014). However, the difference in the degradation of endosperm amyloplast between waxy wheat and non-waxy wheat had rarely been studied.

Although many researchers have studied the special physicochemical properties of waxy wheat, the development and degradation of endosperm amyloplast have been poorly investigated. In this paper, Yangnuo 1 (waxy wheat variety) and Yangmai 13 (nonwaxy wheat variety) were used to investigate the development and degradation of endosperm amyloplast of waxy and non-waxy by using resin semi-thin section technology, histochemical staining technology, frozen section technique and scanning electron microscope observation. 


\section{Materials and Methods}

\section{Plant materials and treatments}

Normal wheat variety Yangmai 13 and waxy wheat variety Yangnuo 1 were provided by the Lixiahe Regional Agricultural Research Institute of Jiangsu Province, China. Field experiment of both two wheat varieties were conducted in the experimental field of Yangzhou University, Jiangsu, China. The date of flowering was recorded accurately by marking florets during the flowering stage.

\section{Morphological and histochemical staining observation of developing wheat}

Wheat caryopses at different days after flowering (DAF) were observed and photographed by using a stereomicroscope (Leica MZ6, Germany) equipped with a digital camera (D3200, Nikon, Thailand). In addition, caryopses at different DAF were cut longitudinally along with the ventral groove. The section was stained by $\mathrm{I}_{2}-\mathrm{KI}\left(3.0 \mathrm{mg} \mathrm{L}^{-1} \mathrm{I}_{2}+3.0\right.$ $\mathrm{mg} \mathrm{L}^{-1} \mathrm{KI}$ ) for $10 \mathrm{~s}$ and observed.

Fresh wheat caryopses at different DAF were cut into 5-mm-thick slices transversely, immersed by embedding medium (Sakura Finetek, USA), cut into $10 \mu \mathrm{m}$-thick slices by freezing microtome (Leica CM1100, Germany), stained with $\mathrm{I}_{2}-\mathrm{KI}\left(3.0 \mathrm{mg} \mathrm{L}^{-1} \mathrm{I}+3.0 \mathrm{mg}\right.$ $\mathrm{L}^{-1} \mathrm{KI}$ ), observed and photographed by using a light microscope (Leica DMLS, Germany) equipped with a digital camera (D3200, Nikon, Thailand).

Determination of soluble sugar, total starch, amylose, amylopectin and 1000-grain weight

The soluble sugar content was determined according to Stitt et al. (1989). The total starch, amylose and amylopectin content were determined according to Zhu et al. (2007) with slight modifications as follows: fresh wheat caryopses at different DAF were processed green removing treatment under $105^{\circ} \mathrm{C}$ for 30 minutes, dried to a constant weight in a ventilated oven under $80{ }^{\circ} \mathrm{C}$ and fully grinded into powder. All tests were repeated at least three times.

\section{Microstructural observation of endosperm amyloplast}

Fresh wheat caryopses at different DAF were cut into $2 \mathrm{~mm}$-thick slices transversely and rapidly immersed into a fixative with $2.5 \%$ glutaraldehyde and $0.05 \mathrm{~mol} \mathrm{~L}^{-1}$ sodium methylarsonate in phosphate buffer $\left(\mathrm{pH} 7.2 ; 0.05 \mathrm{~mol} \mathrm{~L}^{-1}\right)$ at $4{ }^{\circ} \mathrm{C}$ for four hours. The samples were rinsed three times with phosphate buffer, dehydrated by a series of ethanol, displaced by propylene oxide, infiltrated and embeded by low glutinosity resin, and polymerized at $70^{\circ} \mathrm{C}$ for 12 hours in a mould. The polymerized materials were cut into $1 \mu \mathrm{m}$-thick slices by an ultramicrotome (Leica Ultracut R, Germany), stained with $1 \%$ safranin for one minute and $0.01 \mathrm{~mol} \mathrm{~L}^{-1}$ methyl violet for five minutes, observed and 
photographed by using a light microscope (Leica DMLS, Germany) equipped with a digital camera (D3200, Nikon, Thailand).

\section{Size and area determination of endosperm amyloplast}

As showed by Yu et al. (2013), endosperm amyloplasts in images of above experiments were colored by Adobe Photoshop CS5 (Adobe, USA), the size and area of endosperm amyloplasts were counted by Image-Pro Plus (Media Cybernetics, USA). According to above method, the total area was measured, and then the area ratio of amyloplast to endosperm cell was got.

\section{Histochemical staining and microstructural observation of germinating wheat}

After disinfection, wheat seeds were cultivated in an artificial climate chamber according to $\mathrm{Yu}$ et al. (2016). Wheat seeds at different days past germination (DPG) were cut longitudinally along with the ventral groove. The section was stained by $\mathrm{I}_{2}-\mathrm{KI}\left(3.0 \mathrm{mg} \mathrm{L}^{-1} \mathrm{I}_{2}+\right.$ $3.0 \mathrm{mg} \mathrm{L}^{-1} \mathrm{KI}$ ) for $10 \mathrm{~s}$ and observed.

Wheat seeds at different DPG were cut into $2 \mathrm{~mm}$-thick slices transversely and embeded by low glutinosity resin. The slices were stained, observed and photographed as described above.

\section{Ultrastructural observation of endosperm amyloplast during germination}

The wheat starch at different DPG was isolated according to the method of He et al. (2012) with minor modifications. $2 \mathrm{mg}$ of dry starch was dissolved by anhydrous ethanol and dried naturally. The sample was gilded by a sputter coater (BAL-TEC SCD 500, Austria), observed and photographed by an environment scanning electron microscope (SEM) (Philips XL-30 ESEM, Netherlands).

\section{Statistical analysis}

All data were conducted using SPSS Statistics 19.0 (IBM, USA). Fisher's protected least significant difference (LSD) test were used to compare the means at probability significance level $(P<0.05)$. Origin 8.0 (OriginLab, USA) and Adobe Photoshop CS5 were used to make figures.

\section{Results}

\section{Morphological and histochemical staining observation of developing wheat}

The changes in size, shape and color of wheat caryopses from the time of flowering to 40 days after flowering (DAF) are summarized in Fig. S1*. Wheat caryopses had a white

\footnotetext{
*Further details about the Electronic Supplementary Material (ESM) can be found at the end of the article.
} 
surface and colorless syrupy substance within interior at the early stage of development. As development proceeded, the size of caryopses increased, nutritious substances accumulated gradually and the color of surface changed from white to green. At the late stage of development, caryopses were enriched, the shape of caryopses changed no more, and the caryopses turned yellow due to the disintegration of chloroplast. Changes of morphology of waxy and non-waxy wheat varieties were indistinguishable in the process of caryopsis development.

Starch could be stained purple black or reddish-brown by $\mathrm{I}_{2}-\mathrm{KI}$ and the accumulation of starch could be judged by the degree of staining. At the early stage of development, the endosperm could not be stained by $\mathrm{I}_{2}-\mathrm{KI}$, while the pericarp stained purple black because of the existence of amyloplast (Fig. S2). The endosperm started be stained at 8 DAF, the staining area and staining degree became larger and deeper as development proceeded (Fig. S2). The staining degree of the pericarp became slighter and it could not be stained any more due to the degradation of amyloplast (Fig. S3f, h). In general, the pericarp of two wheat varieties could be stained purple black at the early stage of development (Fig. $\mathrm{S} 3 \mathrm{~b}, \mathrm{~d})$. The endosperm of non-waxy wheat was stained purple black by $\mathrm{I}_{2}-\mathrm{KI}$, while that of waxy wheat was stained reddish-brown (Figs S2 and S3).

\section{Soluble sugar, total starch, amylose, amylopectin content and 1000-grain weight of wheat caryopsis}

The soluble sugar content of two wheat varieties was at a high level at the early stage of development, decreased at 8-16 DAF and became stable after 24 DAF (Fig. S4. A). The change of total starch content showed an "S" shaped pattern (Fig. S4. B). The total starch content increased rapidly at 8-16 DAF, the increasing rate slowed down at 16-24 DAF and became stable after 24 DAF. Compared with non-waxy wheat, waxy wheat had a higher soluble sugar content at the early and late stage of development. Waxy wheat had a lower total starch content at the early stage while a higher total starch content at the late stage than non-waxy wheat. Compared with non-waxy wheat, waxy wheat had lower 1000-grain weight, total starch and amylose content, but higher soluble sugar and amylopectin content (Table 1).

Table 1. Content of soluble sugar, total starch, amylose, amylopectin and 1000-grain weight of mature caryopsis

\begin{tabular}{|c|c|c|c|c|c|}
\hline \multicolumn{1}{|c|}{ Varieties } & $\begin{array}{c}\text { 1000-grain } \\
\text { weight (g) }\end{array}$ & $\begin{array}{c}\text { Soluble sugar } \\
\text { content (\%) }\end{array}$ & $\begin{array}{c}\text { Total starch } \\
\text { content (\%) }\end{array}$ & $\begin{array}{c}\text { Amylose } \\
\text { content (\%) }\end{array}$ & $\begin{array}{c}\text { Amylopectin } \\
\text { content (\%) }\end{array}$ \\
\hline Waxy wheat & $41.89 \mathrm{a}$ & $6.24 \mathrm{a}$ & $76.38 \mathrm{a}$ & $0.95 \mathrm{a}$ & $75.43 \mathrm{a}$ \\
\hline Non-waxy wheat & $43.51 \mathrm{~b}$ & $4.37 \mathrm{~b}$ & $79.81 \mathrm{~b}$ & $15.54 \mathrm{~b}$ & $64.27 \mathrm{~b}$ \\
\hline
\end{tabular}

Data in the table are determined from three replicates. Lowercase letters in this table represent significant differences $(P<0.05)$. 


\section{Microstructural observation of endosperm amyloplast}

Few and little endosperm amyloplast appeared firstly at the region adjacent to the nucleus at 6 DAF of two wheat varieties. With the increase of development days, the endosperm cell developed and enriched gradually. Endosperm amyloplast increased in number and size at 8-24 DAF and completed development at 28 DAF (Fig. 1). In this process, the amyloplasts extruded and the nucleus disappeared gradually. There are two kinds of endosperm amyloplast, i.e., large amyloplast and small amyloplast. The large amyloplast appeared earlier and proliferated before $16 \mathrm{DAF}$, while the small amyloplast appeared firstly at 16 DAF (Fig. 1e, f).

In order to analyze the development of endosperm amyloplast, the proportion of small amyloplast and area ratio of amyloplast to endosperm cell were measured (Fig. S5). There were obvious differences in the proportion of small amyloplast between waxy and nonwaxy wheat according to Fig. 1. The small amyloplast proportion of waxy wheat was lower than non-waxy wheat, and less than $60 \%$ of both two wheat varieties at $12 \mathrm{DAF}$. As the development progressed, the proportion of small amyloplast increased gradually. The small amyloplast proportion of waxy wheat was higher than non-waxy wheat, and more than $80 \%$ of both two wheat varieties at 20 DAF. The results indicated that main development stage of small amyloplast of waxy wheat was at 12-16 DAF, and the non-waxy wheat was at 16-20 DAF (Fig. S5A). As the development of endosperm amyloplast, the area ratio of amyloplast to endosperm cell increased gradually, and the ratio of waxy wheat was higher than non-waxy wheat consistently (Fig. S5B). The results indicated that the development of endosperm amyloplast of waxy wheat was better than non-waxy wheat.

\section{Histochemical staining observation of germinating wheat}

As germination progressed, the endosperm changed from hard solid substance to soft gel substance and disappeared eventually (Fig. S6). The endosperm near scutellum was consumed firstly at 2 DPG, and extended to the part away from embryo gradually. Most of the endosperm was depleted and the section almost could not be stained by $\mathrm{I}_{2}-\mathrm{KI}$ at 8 DPG. In general, the degradation of endosperm began from the part close to embryo, and extended to the part away from embryo along with the ventral groove in two wheat varieties. Compared with non-waxy wheat, the degradation rate of endosperm was faster in waxy wheat.

\section{Microstructural and ultrastructural observation of endosperm amyloplast during germination}

In the early stage of wheat seed germination, amyloplast in the endosperm cells were arranged densely and extruded severely (Fig. S7a, d). Amyloplast arranged in loose with larger interspace and the large amyloplast decreased as the germination of seed. Obvious gaps appeared between the endosperm and the aleurone layer at 4 DPG. The large amylo- 
Waxy wheat

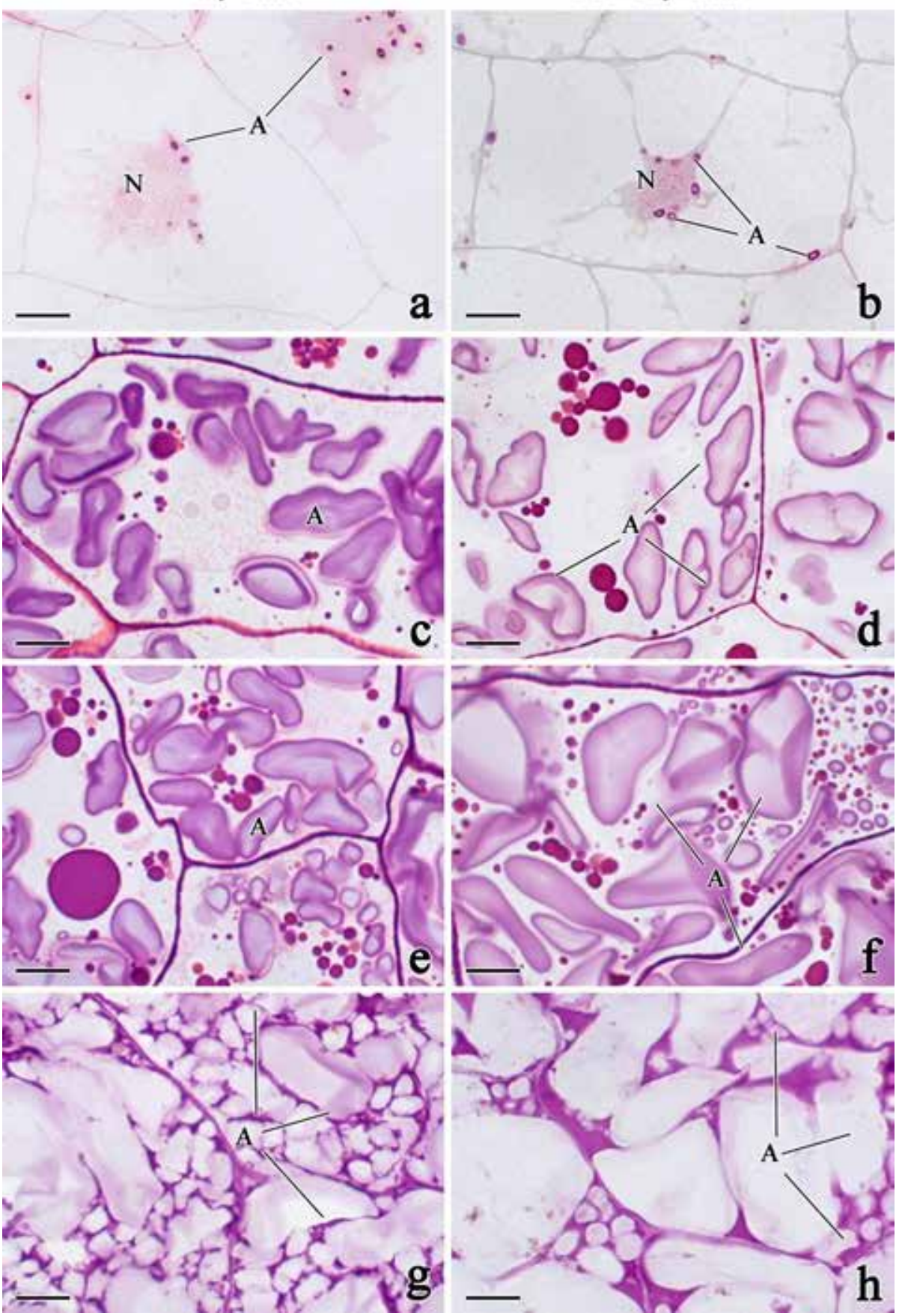

Figure 1. Development of endosperm amyloplast of two wheat varieties. a-b: 6 days after flowering (DAF); c-d: 12 DAF; e-f: 16 DAF; g-h: 32 DAF. A: amyloplast; N: nucleus. Scale bars: $10 \mu \mathrm{m}$ 
plast were hydrolysed into fragments and most of small amyloplast were consumed at 6 DPG. Compared with non-waxy wheat, the hydrolysis rate of endosperm amyloplast was faster in waxy wheat (Fig. S7h, i, k, l).

Few small dissolution pores appeared on the surface of endosperm amyloplast at 2 DPG as shown by the black arrows in Fig. 2. As the germination progressed, the pores became more, bigger and deeper until amyloplast disintegrated to be small amyloplast and be consumed eventually (Fig. 2e, f). On the whole, the degradation rate of endosperm amyloplast in waxy wheat was faster than in non-waxy wheat.

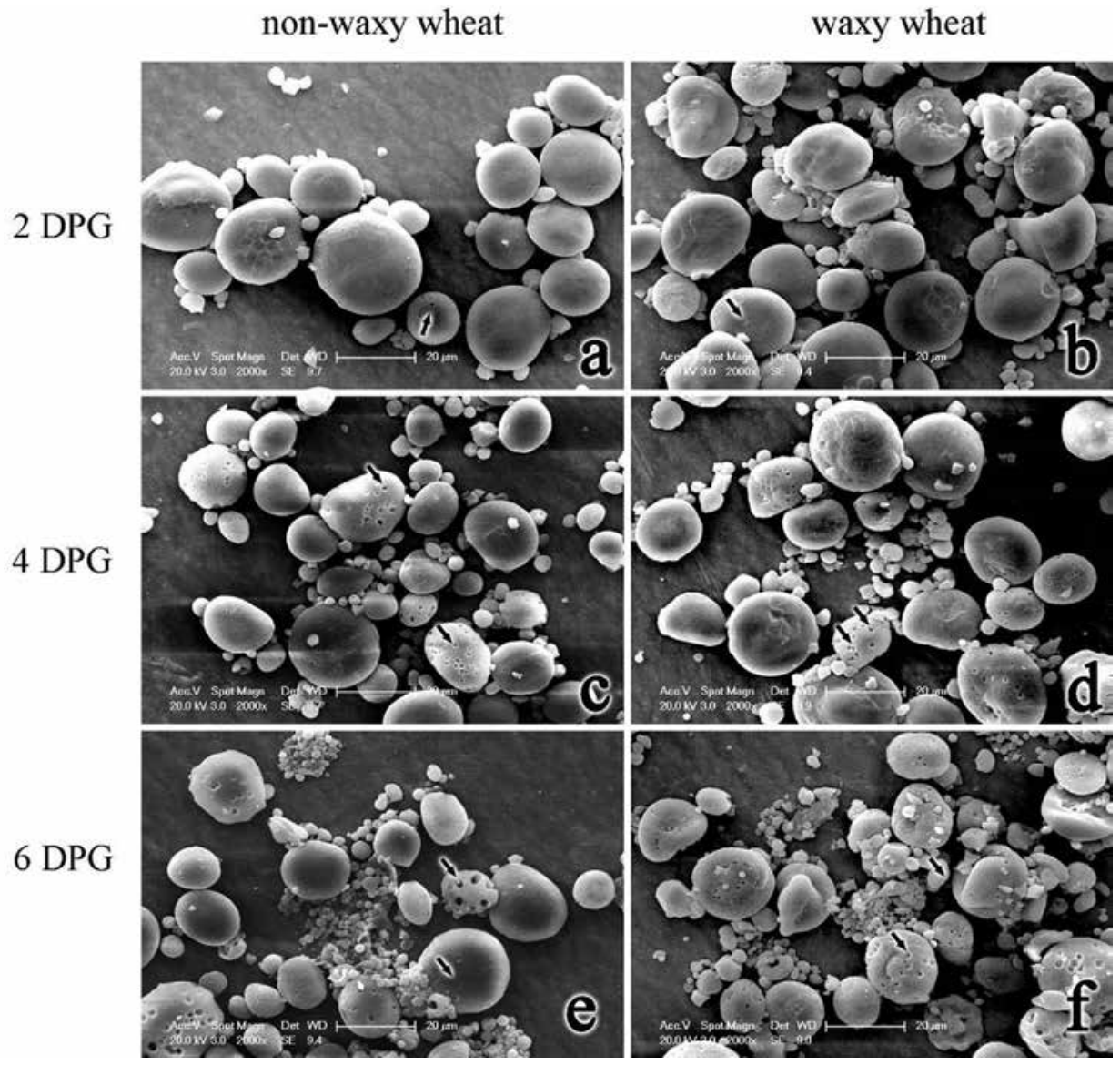

Figure 2. SEM images of endosperm amyloplast of waxy wheat (b, d, f) and non-waxy wheat (a, c, e) at different days past germination (DPG). The black arrows in images indicate dissolution pores 


\section{Discussion}

In the present study the developing endosperm of non-waxy was stained blue-black with $\mathrm{I}_{2}$-KI while the endosperm of waxy wheat was stained reddish-brown possibly due to the lack of the waxy protein, which was also known as granule-bound starch synthase I (GBSSI). However, the pericarp of waxy and non-waxy wheat was stained blue-black possibly because both variety pericarps contain granule-bound starch synthase II (GBSSII) which has an important function in amylose synthesis (Nakamura et al. 1998). The above results indicate that amylose starch synthesis differs between wheat pericarp and endosperm tissues.

In the process of wheat seed germination, the amylase attacked amyloplast and formed many pores on the surface, and the process of hydrolysis went deep into the internal until the amyloplast disintegrated completely. The hydrolysis rate of amyloplast could be influenced by size, integrity, surface-to-volume ratio granule, and amylose to amylopectin ratio (Blazek and Copeland 2010). Furthermore, proteins and lipids on the surface of amyloplast and some hydrolysis products could also slow down the hydrolysis (Colonna et al. 1988; Shin et al. 2004). With the higher surface-to-volume ratio, the small amyloplast was hydrolyzed by $\alpha$-amylase easier than the large amyloplast (Blazek and Copeland 2010; Li et al. 2013). In the present study, the hydrolysis rate of endosperm amyloplast was faster in waxy wheat as the higher proportion of small amyloplast, which was consistent with those studies above.

As the main nutrient reservation organ, the development of endosperm amyloplast directly related to the production and quality of wheat. Though the development of amyloplast has been well characterized, we have little information concerning differences between waxy and non-waxy wheat. In the present study in contrast to non-waxy wheat, endosperm amyloplast of waxy wheat had better development status and the development of small amyloplast in waxy wheat was faster and higher proportion of small amyloplast in mature grain, which was consistent with the findings of Yu et al (Yu et al. 2015) who reported waxy wheat endosperm contained a larger proportion of B-type granules. The development, morphology and size of the endosperm amyloplast related to molecular structure and spatial arrangement of amylopectin (Singh et al. 2010). The high amylopectin content of waxy wheat starch endowed itself special properties and application. Yangnuo 1 , chosen as the waxy wheat variety in the study, only contains $0.95 \%$ amylose content in the wheat grain, this unique trait of waxy wheat confers to a great prospect in industrial areas such as food and starch processing (Zhang H.X et al. 2013). Therefore, the development and application of waxy wheat is becoming more and more important research tasks in modern agricultural fields.

\section{Acknowledgements}

The study was supported by the National Natural Science Foundation (Nos. 31571573, 31171482 , 31270228, 31071341) and a project funded by the Priority Academic Program Development of Jiangsu Higher Education Institutions (PAPD) and the Foundation of Excellent Doctoral Dissertation of Yangzhou University. 


\section{References}

Baik, B.K., Lee, M.R. 2003. Effects of starch amylose content of wheat on textural properties of white salted noodles. Cereal Chem. 80:304-309.

Blazek, J., Copeland, L. 2010. Amylolysis of wheat starches. II. Degradation patterns of native starch granules with varying functional properties. J. Cereal Sci. 52:295-302.

Chao, S., Sharp, P.J., Worland, A.J., Warham, E.J., Koebner, R.M.D., Gale, M.D. 1989. RFLP-based genetic maps of wheat homoeologous group7 chromosomes. Theor. Appl. Genet. 78:495-504.

Colonna, P., Buleon, A., Lemarie, F. 1988. Action of Bacillus subtilis $\alpha$-amylase on native wheat starch. Biotechnol Bioeng. 31:895-904.

Dodson, J.R., Li, X., Zhou, X., Zhao, K., Sun, N., Atahan, P. 2013. Origin and spread of wheat in China. Quaternary Sci. Rev. 72:108-111.

Domínguez, F., Cejudo, F.J. 2014. Programmed cell death (PCD): an essential process of cereal seed development and germination. Front Plant Sci. 5:1-11.

He, J.F., Goyal, R., Laroche, A., Zhao, M.L., Lu, Z.X. 2012. Water stress during grain development affects starch synthesis, composition and physicochemical properties in triticale. J. Cereal Sci. 56:552-560.

Hung, P.V., Maeda, T., Morita, N. 2006. Waxy and high-amylose wheat starches and flours-characteristics, functionality and application. Trends Food Sci. Tech. 17:448-456.

Li, W.H., Shan, Y.L., Xiao, X.L., Luo, Q.G., Zheng, J.M., Ouyang, S.H., Zhang, G.Q. 2013. Physicochemical properties of A- and B- starch granules isolated from hard red and soft red winter wheat. J. Agr. Food Chem. 61:6477-6484.

Ma, H.B., Zhang, X., Wang, C.G., Gao, D.R., Zhang, B.Q., Lv, G.F., Wu, R.L., Cheng, X.M., Wang, X., Cheng, S.H., Bie, T.D. 2013. Effect of $w x$ genes on amylose content, physicochemical properties of wheat starch, and the suitability of waxy genotype for producing Chinese crisp sticks. J. Cereal Sci. 58:140-147.

Macdonald, F.D., Preiss, J. 1985. Partial purification and characterization of granule-bound starch syntheses from normal and waxy maize. Plant Physiol. 78:849-852.

Mira, I., Persson, K., Kurtis, V.V. 2007. On the effect of surface active agents and their structure on the temperature-induced changes of normal and waxy wheat starch in aqueous suspension. Part I. Pasting and calorimetric studies. Carbohyd. Polym. 68:665-678.

Morell, M.K, Rahman, S.L., Abrahams, S., Appels, R. 1995. The biochemistry and molecular biology of starch synthesis in cereals. Aust. J. Plant Physiol. 22:647-660.

Morrison, W.R., Milligan, T.P., Azudin, M.N. 1984. A relationship between the amylose and lipid contents of starches from diploid cereals. J. Cereal Sci. 2:257-271.

Nakamura, T., Yamamori, M., Hirano, H., Hidaka, S., Nagamine, T. 1995. Production of waxy (amylose-free) wheats. Mol. Genet. Genom. 248:253-259.

Nakamura, T., Vrinten, P., Hayakawa, K., Ikeda, J. 1998. Characterization of a granule-bound starch synthase isoform found in the pericarp of wheat. Plant Physiol. 118:451-459.

Parker, M.L. 1985. The relationship between A-type and B-type starch granules in the developing endosperm of wheat. J. Cereal Sci. 3:271-278.

Peña, R.J., Trethowan, R., Pfeiffer, W.H., van Ginkel, M. 2002. Quality (end use) improvement in wheat: compositional, genetic, and environmental factors. J. Crop Prod. 5:1-37.

Sasaki, T., Yasui, T., Matsuki, J. 2000. Effect of amylose content on gelatinization, retrogradation and pasting properties of starches from waxy and nonwaxy wheat and their F1 seeds. Cereal Chem. 77:58-63.

Shin, M., Song, J., Seib, P.A. 2004. In vitro digestibility of cross-linked starches-RS4. Starch/Starke. 56:478483.

Singh, S., Singh, N., Isono, N., Noda, T. 2010. Relationship of granule size distribution and amylopectin structure with pasting, thermal, and retrogradation properties in wheat starch. J. Agr. Food Chem. 58:1180-1188.

Stitt, M., Scheibe, R., Feil, R. 1989. Response of photosynthetic electron transport and carbon metabolism to a sudden decrease of irradiance in the saturating or the limiting range. BBA-Bioenergetics. 973:241-249.

Van Dongen, J.T., Roeb, G.W., Dautzenberg, M., Froehlich, A., Vigeolas, H., Minchin, P.E., Geigenberger, P. 2004. Phloem import and storage metabolism are highly coordinated by the low oxygen concentrations within developing wheat seeds. Plant Physiol. 135:1809-1821. 
Wang, S.J., Wang, J.R., Zhang, W., Li, C.L., Yu, J.L., Wang, S. 2015. Molecular order and functional properties of starches from three waxy wheat varieties grown in China. Food Chem. 181:43-50.

Yu, X.R., Zhou, L., Jing, Y.P., Liu, D.T., Hu, M.L., Xiong, F., Wang, Z. 2013. Application of image-pro plus in analysis of wheat starch granule microscopic image. Journal of Chinese Electron Microscopy Society. 32:344-351. (In Chinese with English abstract)

Yu, X.R., Yu, H., Zhang, J., Shao, S.S., Zhou, L., Xiong, F., Wang, Z. 2015. Comparison of endosperm starch granule development and physiochemical properties of starches from waxy and non-waxy wheat. Int. J. Food Prop. 18:2409-2421.

Zhang H.X., Zhang W, Xu C.Z., Zhou, X. 2013. Morphological features and physicochemical properties of waxy wheat starch. Int. J. Biol. Macromol. 62:304-309.

Zhu, T., Jackson, D.S., Wehling, R.L., Geera, B. 2007. Comparison of amylose determination methods and the development of a dual wavelength iodine binding technique. Cereal Chem. 85:51-58.

\section{Electronic Supplementary Material (ESM)}

Electronic Supplementary Material (ESM) associated with this article can be found at the website of CRC at http://www.akademiai.com/content/120427/

Electronic Supplementary Figure S1. Stereomicroscope images of caryopses profile of waxy wheat (A) and non-waxy wheat (B). The numbers in images indicate days after flowering (DAF). Scale bars: $2 \mathrm{~mm}$

Electronic Supplementary Figure S2. Stereomicroscope images of vertical sections of waxy wheat (A) and non-waxy wheat $(B)$ caryopses stained by $\mathrm{I}_{2}-\mathrm{KI}$. The numbers in images indicate days after flowering (DAF). Scale bars: $2 \mathrm{~mm}$

Electronic Supplementary Figure S3. Different dyeing properties of amyloplast from endosperm cell and pericarp. a-h: frozen section stained by $\mathrm{I}_{2}-\mathrm{KI}$; a-d: 8 days after flowering (DAF); e-h: 16 DAF. A: amyloplast; EC: endosperm cell; Pe: pericarp. Scale bars: $50 \mu \mathrm{m}$

Electronic Supplementary Figure S4. Changes of soluble sugar (A) and total starch content (B) during development

Electronic Supplementary Figure S5. Changes of proportion of small amyloplast (A) and area ratio of amyloplast to endosperm cell (B) during development. Lowercase letters in this figure represent significant differences $(P<0.05)$

Electronic Supplementary Figure S6. Stereomicroscope images of vertical sections of waxy wheat and non-waxy wheat caryopses stained by $\mathrm{I}_{2}-\mathrm{KI}$. AL: aleurone layer; Em: embryo; En: endosperm; Sc: scutellum;

VG: ventral groove. Scale bars: $2 \mathrm{~mm}$

Electronic Supplementary Figure S7. Degradation of endosperm (a-f) and amyloplast (g-1) during germination. A: amyloplast; AL: aleurone layer; En: endosperm; Pe: pericarp. Scale bars: $50 \mu \mathrm{m}(\mathrm{a}-\mathrm{f}) ; 10 \mu \mathrm{m}(\mathrm{g}-1)$ 